\title{
Is Ultracision Knife Safe and Efficient for Breast Capsulectomy? A Preliminary Study
}

\author{
Mathias Tremp • Pietro G. di Summa • Dominique Schaakxs • \\ Ulrich Rieger · Wassim Raffoul • Dirk J. Schaefer • \\ Daniel F. Kalbermatten
}

Received: 27 November 2011/Accepted: 2 March 2012/Published online: 27 April 2012

(C) Springer Science+Business Media, LLC and International Society of Aesthetic Plastic Surgery 2012

\begin{abstract}
Background Silicone breast implants are used to a wide extent in the field of plastic surgery. However, capsular contracture remains a considerable concern. This study aimed to analyze the effectiveness and applicability of an ultracision knife for capsulectomy breast surgery.

Methods A prospective, single-center, randomized study was performed in 2009. The inclusion criteria specified female patients 20-80 years of age with capsular contracture (Baker 3-4). Ventral capsulectomy was performed using an ultracision knife on one side and the conventional Metzenbaum-type scissors and surgical knife on the collateral side of the breast. Measurements of the resected capsular ventral fragment, operative time, remaining breast tissue, drainage time, seroma and hematoma formation, visual analog scale pain score, and sensory function of the nipple-areola complex were assessed. In addition, histologic analysis of the resected capsule was performed.

Results Five patients (median age, 59.2 years) were included in this study with a mean follow-up period of 6 months. Three patients had Baker grade 3 capsular contracture, and two patients had Baker grade 4 capsular
\end{abstract}

M. Tremp · D. J. Schaefer · D. F. Kalbermatten $(\bowtie)$

Department of Plastic, Reconstructive, Aesthetic, and Hand Surgery, University Hospital Basel,

Spitalstrasse 21, 4031 Basel, Switzerland

e-mail: dkalbermatten@uhbs.ch

P. G. di Summa · D. Schaakxs · W. Raffoul ·

D. F. Kalbermatten

Division of Plastic, Reconstructive, and Aesthetic Surgery

CHUV, University Hospital of Lausanne, Lausanne, Switzerland

U. Rieger

Department of Plastic, Reconstructive, and Aesthetic Surgery,

University Innsbruck, Innsbruck, Austria contracture. The ultracision knife was associated with a significantly lower pain score, shorter operative time, smaller drainage volume, and shorter drainage time and resulted in a larger amount of remaining breast tissue. Histologic analysis of the resected capsule showed no apoptotic cells in the study group or control group.

Conclusions The results suggest that ventral capsulectomy with Baker grade 3 or 4 contracture using the ultracision knife is feasible, safe, and more efficient than blunt dissection and monopolar cutting diathermy and has a short learning curve.

Level of Evidence II This journal requires that authors assign a level of evidence to each article. For a full description of these Evidence-Based Medicine ratings, please refer to the Table of Contents or the online Instructions to Authors at www.springer.com/00266.

Keywords Comparative effectiveness research . Feasibility study $\cdot$ Fibrosis $\cdot$ Silicone gels

Silicone breast implants have been used to a wide extent in aesthetic and reconstructive surgery. However, the development of capsular contracture remains the most significant drawback. The degree of capsular contraction is clinically assessed and assigned to one of four Baker classification grades [28], which range from an impalpable and invisible shell (grade 1) to a very firm shell with implant dislocation and deformity (grade 4) [20].

The exact etiology of capsular contracture still is not clear, but two hypotheses exist, positing either a nonspecific inflammatory process directed against the foreign body or a periprosthetic bacterial contamination [12, 20, $22,23]$. More is known about the incidence and the position of the prostheses. For example, submuscular rather 
than subglandular placement is a well-established method for decreasing the contracture rate [5, 29], probably due to the massaging action of the overlying pectoralis major muscle [27].

The incidence of symptomatic capsular contracture can range from 4 to $74 \%$ [21], but large-scale studies report an overall prevalence of $8-17 \%[9,13]$.

Patients with capsular contraction experience hardness and firmness of the mammary area, discomfort and pain with displacement, or palpability of the implants leading to cosmetic and functional failure [1, 22]. A relationship between the number of myofibroblasts, the tensile strength of the breast implant capsule, and the degree of breast capsular contracture has been reported [10].

In general, a complete capsulectomy should be performed when a replacement implant is inserted along a different tissue plane than the explanted one when no implant will be reinserted after explantation or when a pathologic capsule is present [3]. Closed capsulotomy may not be advisable given the risk of implant migration and hematoma or rupture with extracapsular silicone extravasation [6]. Findings have shown that total capsulectomy for subglandular silicone breast implant capsular contracture is superior to anterior disc capsulectomy, with a lower recurrence rate [6]. However, anterior capsulectomy is simpler to perform and associated with a lower morbidity rate $[2,6]$.

In a small series by Xue and Lee [30], breast implants were removed and repositioned anterior to the primary capsule in patients with Baker grade 3 or 4 capsular contracture. For implant-based breast reconstruction, the inframammary fold represents one of the most important anatomic landmarks, and if it is sacrificed or dislocated cranially or caudally, it needs to be recreated [2].

To date, capsulectomies are performed using a combination of blunt dissection and monopolar cutting diathermy. However, both techniques have the disadvantages of considerable morbidity, long operative time, and high blood loss. Recently, ultrasonic instruments have emerged as an alternative to electrocautery for surgical dissection and hemostasis. The ultracision knife is a cutting and coagulating surgical device used worldwide for both endoscopic and open surgical procedures [15, 18, 26]. Vibrating at $55 \mathrm{kHz}$, the blade provides both cutting and coagulation with minimal lateral thermal damage, reducing the potential for damage to adjacent nerve structures [24]. Because these devices are driven by ultrasound rather than electrical energy, no electricity is passed to or through the patient.

This preliminary study aimed to evaluate the safety and feasibility of an ultrasonic knife (Harmonic Synergy Curved Blade, SNGCB; Ethicon Endo-Surgery, LLC, Norderstedt, Germany) used in capsulectomy for Baker grade 3 or 4 capsular contracture.

\section{Materials and Methods}

A prospective, single-center randomized study was performed in 2009. The study was approved by the local institutional review board, including the patient's full consent according to the Helsinki guidelines. The inclusion criteria specified female patients 20-80 years of age with capsular contracture (Baker grade 3 or 4) after breast augmentation or reconstruction. Written informed consent was obtained from each patient. The exclusion criteria ruled out patients receiving medical treatment-type chemotherapy or cortisone and patients with diabetes, skin eczema, or vasculitis. Medical history, comorbidities (elevated body mass index [BMI], diabetes mellitus, tobacco abuse, hypertension), preoperative medications, and preoperative sensory function of the nipple-areola complex according to Semmes-Weinstein filament (1 g) testing were assessed.

\section{Surgical Technique}

Surgery was performed with the patient under general anesthesia using an inframammary approach. Prophylactic antibiotics were given, and all procedures were performed by the senior author (D.K.) either personally or in a supervisory capacity. For ventral capsulectomy and breast augmentation, the ultracision knife (Harmonic Synergy Curved Blade, SNGCB; Ethicon Endo-Surgery, LLC, Norderstedt, Germany) was used on the study side of the breast, and the conventional Metzenbaum-type scissors and a surgical knife were used on the collateral side. This provided a high level of comparison matching because each patient acted as both a study subject and a control subject. The pockets and new implants were routinely irrigated with saline, and all breasts were drained.

\section{Clinical Outcome Measures}

\section{Intraoperative and Early Postoperative Outcome}

Intraoperatively, with the aim of preserving the largest amount of breast tissue possible, measurements of the resected capsular ventral fragment (length, size, thickness) and operative time were determined. The remaining breast tissue was measured by calipometry at the level of the nipple-areola complex. After 3 postoperative days, the following were evaluated: drainage time as well as seroma and hematoma formation by volume in drains. The visual analog scale (VAS) pain scores for the control and study sides during the first 3 days also were determined. In addition, histologic assessment of the resected capsule for apoptotic cells due to thermal damage was performed. 

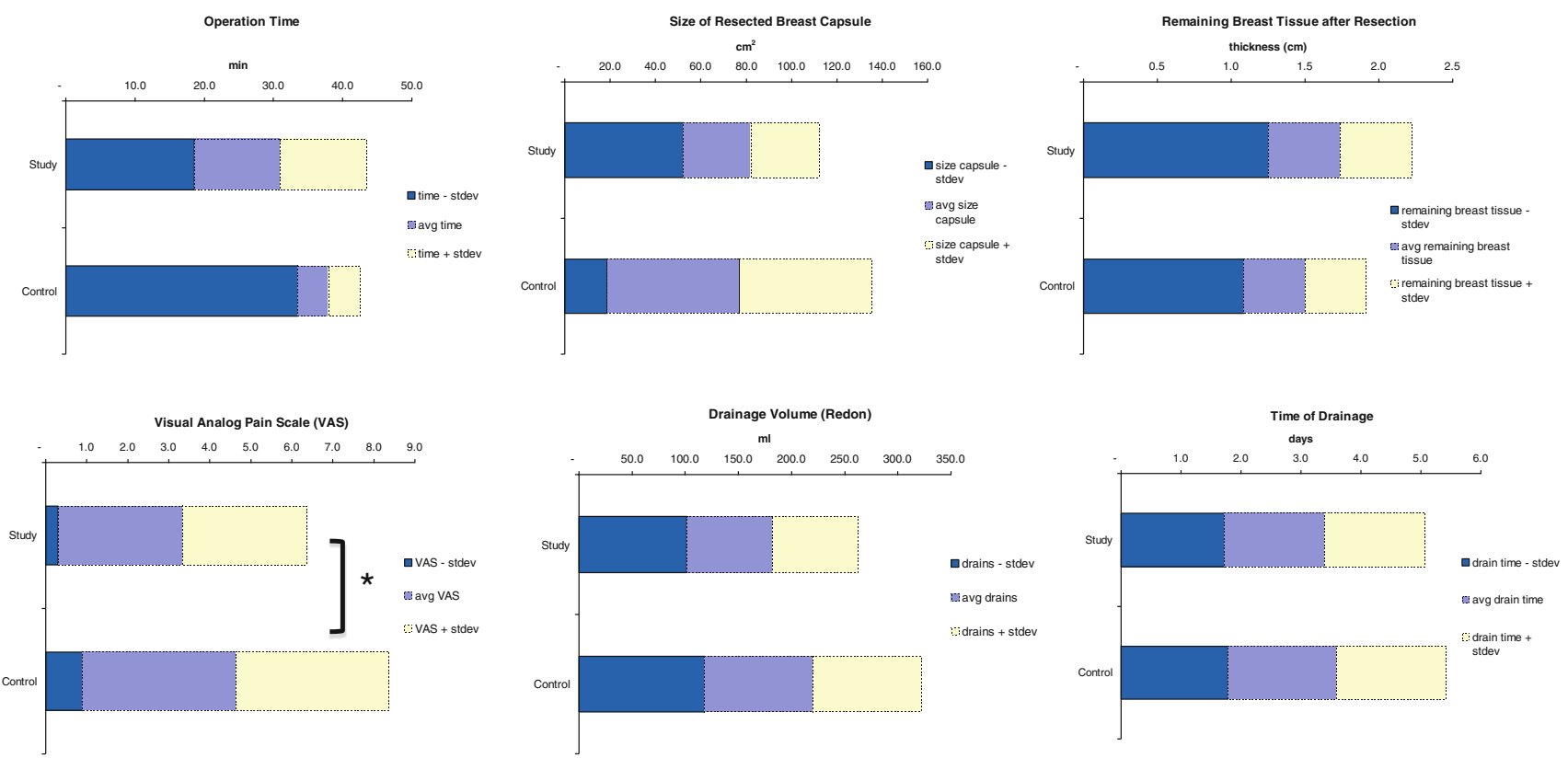

$\mathrm{N}=5$ patients

Fig. 1 Evaluation of five surgical patients who underwent use of the ultracision knife shows a trend toward a better outcome in six categories. Ultracision $=$ study. Scissors $/$ monopolar coagulation $=$ control. $* p<0.05$

\section{Late Postoperative Outcome}

After 6 months, the sensory function of the nipple-areola complex was assessed by Semmes-Weinstein monofilament testing $(1 \mathrm{~g})$. Late postoperative complications were assessed at the same time.

\section{Statistical Analysis}

The data are expressed as mean \pm standard error of the mean. The independent Student's $t$ test was used to compare the means between the two groups using Microsoft Excel (Microsoft Corp., Redmond, USA). A $p$ value less than 0.05 was considered to be statistically significant. Correlations between the two groups also were calculated. The following convention was used in the figures: ${ }^{*} p<0.05$.

\section{Results}

The study enrolled five patients ranging in age from 55 to 64 years (median, 59.2 years). Three patients had Baker grade 3 capsular contracture, and two patients had Baker grade 4 capsular contracture on both sides. The follow-up period was 6 months, and no serious adverse events occurred during the study period. Preoperatively, three patients had intact sensation of the nipple-areola complex on both sides, whereas postoperatively, only two patients were found to have normal sensation on both sides, according to the Semmes-Weinstein test (1 g).
In the early postoperative period, one patient experienced bilateral hematoma, which was treated conservatively. This patient also had diminished sensation of the nipple-areola complex on both sides postoperatively. No late postoperative complications occurred in this study. As seen in Fig. 1, the ultracision knife showed a trend toward a better outcome compared with conventional scissors and surgical knife.

The mean operative time was $31 \pm 12.4 \mathrm{~min}$ in the study group and $38 \pm 4.5 \mathrm{~min}$ in the control group (correlation, $0.16 ; p=0.28)$. The capsular size was $82 \pm 29.9 \mathrm{~cm}^{2}$ in the study group and $77.2 \pm 58.4 \mathrm{~cm}^{2}$ in the control group (correlation, $0.91 ; p=0.75$ ), whereas the thickness of the capsule was $0.7 \pm 0.4 \mathrm{~cm}$ in the study group and $0.5 \pm 0.6 \mathrm{~cm}$ in the control group (correlation, $0.68 \% ; p=0.35)$.

A representative patient is shown in Figs. 2 and 3. There is an obvious size difference between ventral capsulectomy with the ultracision knife and surgery using conventional surgical knife/scissors, with the capsule remaining intact after use of the ultracision knife.

The thickness of the remaining breast tissue after resection was $1.7 \pm 0.5 \mathrm{~cm}$ in the study group and $1.5 \pm 0.4 \mathrm{~cm}$ in the control group (correlation, 0.81; $p=0.14)$. The same trend was seen in pain assessment. The VAS was $2.8 \pm 3.0$ in the study group and $4.6 \pm 3.7$ in the control group (correlation, 0.95; $p=0.03$ ). In addition, the study group had less volume in the drains $(182 \pm 80.7 \mathrm{~mL})$ than the control group $(220 \pm 102 \mathrm{~mL})$ 
Fig. 2 a Representative case of a patient with grade 3 capsular contracture. b Partial capsulectomy and implant removal was performed on the left side with the ultracision knife and on the right side with the conventional surgical knife and scissors. c Size difference after ventral capsulectomy. d The capsule remains intact after use of the ultracision knife
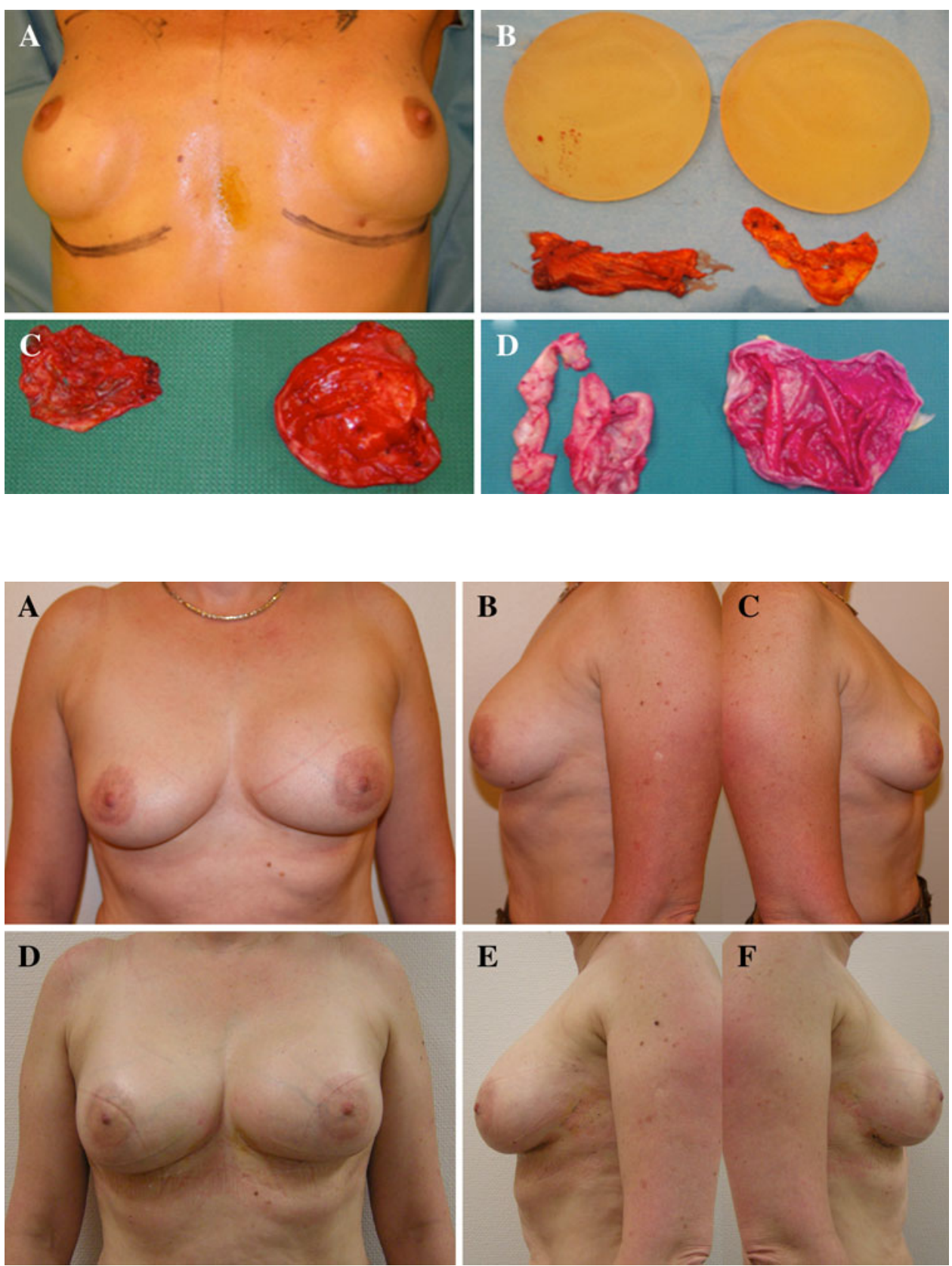

Fig. 3 Photo documentation of a representative patient with grade 3 capsular contracture before (a-c) and after (df) partial capsulectomy and implant removal with the ultracision knife on the left side and the conventional surgical knife and scissors on the right side (correlation, $0.47 ; p=0.42$ ). The drain time was found to be shorter in the study group $(3.4 \pm 1.7$ vs. $3.6 \pm$ 1.8 days) (correlation, $0.97 ; p=0.37$ ). Histologic analysis of the resected capsule showed no apoptotic cells in the study group or the control group.

\section{Discussion}

This study showed that ventral capsulectomy with the ultracision knife leads to a shorter operative time, greater capsule resection volume, and more remaining breast tissue (likely due to a more precise dissection of the capsule) and results in significantly less pain to the patient. In addition, drain time and drain volume were less in the study group. Assessment of the nipple-areola complex showed no difference between the study and control groups, so sensory changes cannot be attributed to a specific method. Also, no difference in histologic analysis was found between the two methods.

We performed ventral capsulectomy because of its lower morbidity compared with posterior capsulectomy, which can injure the intercostal muscles and vasculature, causing further complications, such as pneumothorax [16]. 


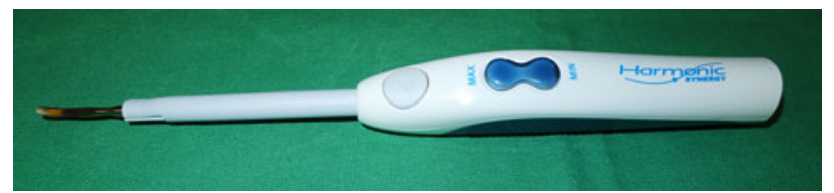

Fig. 4 Ultracision knife (Harmonic scalpel provided by Ethicon Endo-Surgery, LLC, Norderstedt, Germany [Europe] GmbH)

We presumed that the ultracision knife would have fewer side effects in posterior capsulectomy than blunt dissection and monopolar cutting diathermy, as with ventral capsulectomy, and that the results could be comparable with those of total capsulectomy.

Handling of the ultracision knife (Fig. 4) was described as superior by the surgeons involved in this study because the device has a smooth and almost bloodless cutting of the capsule but not the silicone implant. In addition, our study design that used the ultracision knife on one side and the conventional Metzenbaum-type scissors and surgical knife on the collateral side of the breast allowed a high level of comparison because the same patient served as both a study subject and a control subject. A similar study design and level of evidence have been reported previously $[11,19]$.

Little data exist regarding the effectiveness of the ultracision knife in capsulectomy for capsular contracture. However, studies in other fields describing the feasibility and morbidity of the ultracision knife do exist, and our results mirror these studies $[4,7,8,17,25]$. Ceccaldi et al. [4] evaluated the ultrasonic energy dissection technique in breast reconstruction with the autologous latissimus dorsi flap in a prospective monocentric study involving 21 patients. In their study, no blood transfusion was necessary, and the authors concluded that the ultrasonic energy dissection technique in breast reconstruction had no additional disadvantages and resulted in less seroma formation than the conventional scalpel [4].

In another study, the ultracision knife was compared with electrocautery for performing modified radical mastectomy [8]. The authors found a significantly smaller drainage volume and blood loss in the ultracision knife group, with a significant reduction in drain days, and concluded that modified radical mastectomy using the ultracision knife was feasible and had a short learning curve [8]. The ultracision knife also was found to reduce the onset of arm lymphedema in sentinel node biopsy with axillary node sampling [17] and resulted in less blood loss, fewer draining days, a lower seroma rate, and a smaller drainage volume in axillary dissection than electrocautery [25].

Deo and Shukla [7] evaluated the feasibility of using ultrasonic energy for modified radical mastectomy. Compared with scalpel and electrocautery, the authors found a reduction in blood loss and drainage volume using the ultracision knife. None of the 14 patients experienced flap necrosis or hematoma, and a seroma developed in only one patient.

In contrast to these findings, one prospective randomized study involving 32 patients evaluated the effectiveness of the ultracision knife in breast surgery [14]. There was no significant reduction in seroma formation, wound complications, or pain compared with electrocautery, and the authors concluded that use of the ultracision knife offered no significant clinical advantage or cost benefit.

This study had some limitations. The number of patients was too small for any definitive conclusions to be drawn, and the study is therefore considered a preliminary study. Also, the follow-up period was too short, and the recurrence rate for both groups is thus reported after only 1 year. Large-scale, prospective, randomized trials comparing the ultracision knife with conventional techniques during a longer follow-up period are needed.

\section{Conclusions}

In summary, our results suggest that ventral capsulectomy for a Baker grade 3 or 4 contracture using the ultracision knife is feasible, safe, and more efficient than blunt dissection and monopolar cutting diathermy and has a short learning curve. Thus, it would be worthwhile to perform larger studies using the ultracision knife to evaluate its use in more depth.

Acknowledgments We thank Dr. Daniel Haselbach for preparation of the ethical commission submission. We thank Prof. Gerhard Pierer, MD, and Martin Haug, MD, University of Basel, for administrative support. Material for this study was kindly provided by Ethicon EndoSurgery $\mathrm{GmbH}$, Norderstedt, Germany. The Harmonic Synergy Curved Blade (SNGCB; Ethicon Endo-Surgery LLC, USA) was used in this study.

\section{References}

1. Barnsley GP, Sigurdson LJ, Barnsley SE (2006) Textured surface breast implants in the prevention of capsular contracture among breast augmentation patients: a meta-analysis of randomized controlled trials. Plast Reconstr Surg 117:2182-2190

2. Bogetti P, Cravero L, Spagnoli G, Devalle L, Boriani F, Bocchiotti MA, Renditore S, Baglioni E (2007) Aesthetic role of the surgically rebuilt inframammary fold for implant-based breast reconstruction after mastectomy. J Plast Reconstr Aesthet Surg 60:1225-1232

3. Bracaglia R, Fortunato R, Gentileschi S (2008) A simple technique to make breast implant capsulectomy easier. J Plast Reconstr Aesthet Surg 61:1550-1551

4. Ceccaldi PF, Ducarme G, Kere D, Wernert R (2006) Effect of ultrasonic energy dissection technique in breast reconstruction with the autologous latissimus dorsi flap. J Gynecol Obstet Biol Reprod Paris 35:762-766 
5. Collis N, Coleman D, Foo IT, Sharpe DT (2000) Ten-year review of a prospective randomized controlled trial of textured versus smooth subglandular silicone gel breast implants. Plast Reconstr Surg 106:786-791

6. Collis N, Sharpe DT (2000) Recurrence of subglandular breast implant capsular contracture: Anterior versus total capsulectomy. Plast Reconstr Surg 106:792-797

7. Deo SV, Shukla NK (2000) Modified radical mastectomy using harmonic scalpel. J Surg Oncol 74:204-207

8. Deo SV, Shukla NK, Asthana S, Niranjan B, Srinivas G (2002) A comparative study of modified radical mastectomy using harmonic scalpel and electrocautery. Singap Med J 43:226-228

9. Henriksen TF, Fryzek JP, Holmich LR, McLaughlin JK, Kjoller K, Hoyer AP, Olsen JH, Friis S (2005) Surgical intervention and capsular contracture after breast augmentation: a prospective study of risk factors. Ann Plast Surg 54:343-351

10. Hwang K, Sim HB, Huan F, Kim DJ (2010) Myofibroblasts and capsular tissue tension in breast capsular contracture. Aesthetic Plast Surg 34:716-721

11. Jones BM, Grover R, Hamilton S (2007) The efficacy of surgical drainage in cervicofacial rhytidectomy: a prospective, randomized, controlled trial. Plast Reconstr Surg 120:263-270

12. Khan UD (2010) Breast augmentation, antibiotic prophylaxis, and infection: comparative analysis of 1,628 primary augmentation mammoplasties assessing the role and efficacy of antibiotics prophylaxis duration. Aesthetic Plast Surg 34:42-47

13. Kjoller K, Holmich LR, Jacobsen PH, Friis S, Fryzek J, McLaughlin JK, Lipworth L, Henriksen TF, Jorgensen S, Bittmann S, Olsen JH (2002) Epidemiological investigation of local complications after cosmetic breast implant surgery in Denmark. Ann Plast Surg 48: 229-237

14. Kontos M, Kothari A, Hamed H (2008) Effect of harmonic scalpel on seroma formation following surgery for breast cancer: a prospective randomized study. J BUON 13:223-230

15. Langer C, Markus P, Liersch T, Fuzesi L, Becker H (2001) UltraCision or high-frequency knife in transanal endoscopic microsurgery (TEM)? Advantages of a new procedure. Surg Endosc 15:513-517

16. Lee HK, Jin US, Lee YH (2011) Subpectoral and precapsular implant repositioning technique: correction of capsular contracture and implant malposition. Aesthetic Plast Surg 35:1126-1132

17. Lumachi F, Basso SM, Bonamini M, Marino F, Marzano B, Milan E, Waclaw BU, Chiara GB (2009) Incidence of arm lymphoedema following sentinel node biopsy, axillary sampling, and axillary dissection in patients with breast cancer. In Vivo 23:1017-1020

18. Morino M, Rimonda R, Allaix ME, Giraudo G, Garrone C (2005) Ultrasonic versus standard electric dissection in laparoscopic colorectal surgery: a prospective randomized clinical trial. Ann Surg 242:897-901 (discussion 901)

19. Oliver DW, Hamilton SA, Figle AA, Wood SH, Lamberty BG (2001) A prospective, randomized, double-blind trial of the use of fibrin sealant for face-lifts. Plast Reconstr Surg 108:2101-2105 (discussion 2106-2107)

20. Poeppl N, Schreml S, Lichtenegger F, Lenich A, EisenmannKlein M, Prantl L (2007) Does the surface structure of implants have an impact on the formation of a capsular contracture? Aesthetic Plast Surg 31:133-139

21. Prantl L, Angele P, Schreml S, Ulrich D, Poppl N, EisenmannKlein M (2006) Determination of serum fibrosis indexes in patients with capsular contracture after augmentation with smooth silicone gel implants. Plast Reconstr Surg 118:224-229

22. Prantl L, Schreml S, Fichtner-Feigl S, Poppl N, Eisenmann-Klein M, Schwarze H, Fuchtmeier B (2007) Clinical and morphological conditions in capsular contracture formed around silicone breast implants. Plast Reconstr Surg 120:275-284

23. Rieger UM, Pierer G, Luscher NJ, Trampuz A (2009) Sonication of removed breast implants for improved detection of subclinical infection. Aesthetic Plast Surg 33:404-408

24. Salami A, Dellepiane M, Bavazzano M, Crippa B, Mora F, Mora $R$ (2008) New trends in head and neck surgery: A prospective evaluation of the Harmonic scalpel. Med Sci Monit 14:PI1-PI15

25. Sanguinetti A, Docimo G, Ragusa M, Calzolari F, D'Ajello F, Ruggiero R, Parmeggiani D, Pezzolla A, Procaccini E, Avenia N (2010) Ultrasound scissors versus electrocautery in axillary dissection: our experience. G Chir 31:151-153

26. Schmidbauer S, Hallfeldt KK, Sitzmann G, Kantelhardt $T$, Trupka A (2002) Experience with ultrasound scissors and blades (UltraCision) in open and laparoscopic liver resection. Ann Surg 235:27-30

27. Siggelkow W, Klosterhalfen B, Klinge U, Rath W, Faridi A (2004) Analysis of local complications following explantation of silicone breast implants. Breast 13:122-128

28. Spear SL, Baker JL Jr (1995) Classification of capsular contracture after prosthetic breast reconstruction. Plast Reconstr Surg 96:1119-1123 (discussion 1124)

29. Wong CH, Samuel M, Tan BK, Song C (2006) Capsular contracture in subglandular breast augmentation with textured versus smooth breast implants: a systematic review. Plast Reconstr Surg 118:1224-1236

30. Xue H, Lee SY (2011) Correction of capsular contracture by insertion of a breast prosthesis anterior to the original capsule and preservation of the contracted capsule: technique and outcomes. Aesthetic Plast Surg 35:1056-1060 